\title{
Knowledge mobilization tool to promote, protect, and support breastfeeding during COVID-19
}

\author{
Shela Akbar Ali Hirani ${ }^{1}$ (I) $\cdot$ Megan Pearce $^{1} \cdot$ Amanda Lanoway $^{2}$ \\ Received: 23 October 2020 / Accepted: 20 April 2021 / Published online: 21 May 2021 \\ (C) The Canadian Public Health Association 2021
}

\begin{abstract}
Setting This knowledge mobilization project was conceptualized to increase awareness among breastfeeding mothers and the general public on safe infant feeding practices during the COVID-19 pandemic by addressing myths and misconceptions associated with breastfeeding practices, guiding breastfeeding mothers to make informed decisions around child feeding practices, and offering meaningful guidance in simple language through a short online animated video.

Intervention This project was undertaken in four phases. During phase 1, an informal discussion was held with the breastfeeding mothers, service providers, and community partner in identifying issues surrounding lactation counselling facilities during the COVID-19 pandemic. During phase 2, recommendations from 23 organizations with regard to breastfeeding during COVID-19 were reviewed and analyzed. During phase 3, using evidence from reliable sources, a 5-minute animated e-resource on breastfeeding during COVID-19 was conceptualized and developed. During phase 4, the e-resource was disseminated to the breastfeeding mothers, general public, post-secondary institutions, and organizations providing services to breastfeeding mothers in Canada.

Outcomes This evidence-based e-resource facilitated addressing misconceptions around breastfeeding during COVID-19 and raising public awareness on safe infant feeding practices during this pandemic. Overall, the video was described as an informative, user-friendly, useful, and easily accessible resource by breastfeeding mothers who were in self-isolation with little access to healthcare services during the pandemic.

Implications This project highlighted the importance of patient engagement and collaboration with the community partner in protecting breastfeeding during the COVID-19 pandemic. It further illustrated how informational e-resources can protect breastfeeding in situations where breastfeeding mothers' access to healthcare services is compromised.
\end{abstract}

\section{Résumé}

Lieu Notre projet de mobilisation des connaissances vise à sensibiliser les mères allaitantes et le grand public aux pratiques d'alimentation sûres pour les nourrissons durant la pandémie de COVID-19 en abordant les mythes et les idées fausses associés aux pratiques d'allaitement maternel, en aidant les mères allaitantes à prendre des décisions éclairées quant aux pratiques d'alimentation des bébés et en offrant des conseils utiles, en langage simple, dans une courte vidéo animée accessible en ligne. Intervention Le projet a été mené en quatre phases. Pendant la phase 1, nous avons eu une discussion informelle avec les mères allaitantes, les dispensateurs de services et le partenaire associatif pour définir les problèmes entourant les servicesconseils sur la lactation durant la pandémie de COVID-19. Pendant la phase 2, nous avons vu et analysé les recommandations de 23 organismes concernant l'allaitement durant la COVID-19. Pendant la phase 3, à l'aide de données probantes provenant de sources fiables, nous avons conceptualisé et créé une cyberressource animée de cinq minutes sur l'allaitement durant la COVID-19. Pendant la phase 4 , nous avons diffusé cette cyberressource aux mères

Shela Akbar Ali Hirani

shela.hirani@uregina.ca

1 Faculty of Nursing, University of Regina, 3737 Wascana Parkway, Regina, Saskatchewan S4S 0A2, Canada

2 United Way Regina, 1440 Scarth Street, Regina, Saskatchewan S4R 2E9, Canada 
allaitantes, au grand public, à des établissements d'enseignement postsecondaires et à des organismes de services aux mères allaitantes au Canada.

Résultats Cette cyberressource factuelle a permis d'aborder plus facilement les idées fausses entourant l'allaitement durant la COVID-19 et de sensibiliser le public aux pratiques sûres d'alimentation des nourrissons durant la pandémie. Dans l'ensemble, des mères allaitantes en isolement, qui avaient peu accès aux services de soins de santé durant la pandémie, ont trouvé notre vidéo informative, conviviale, utile et facilement accessible.

Conséquences Ce projet souligne l'importance du contact avec la patiente et de la collaboration avec le partenaire associatif pour protéger l'allaitement maternel durant la pandémie. Il montre aussi que des ressources informationnelles accessibles en ligne peuvent protéger l'allaitement dans les situations où les mères allaitantes ont moins accès aux services de soins de santé.

Keywords Breastfeeding $\cdot$ COVID-19 $\cdot$ E-resource $\cdot$ Informational support $\cdot$ Mothers $\cdot$ Canada

Mots-clés Allaitement au sein $\cdot$ COVID-19 $\cdot$ cyberressources $\cdot$ soutien informationnel $\cdot$ mères $\cdot$ Canada

\section{Introduction}

COVID-19 has produced devastating economic, social, and mental health burdens on people worldwide (Acter et al., 2020). COVID-19 is reported to have a different impact on women than on men due to women's often central role in caring for the family (Wenham et al., 2020). The COVID-19 pandemic has increased the vulnerability of mothers and young children due to lack of opportunity to have regular contact with healthcare professionals, inaccessibility to breastfeeding counselling facilities, growing myths and misconceptions around breastfeeding during the COVID-19 pandemic, loss of family income, and stress associated with an uncertain future. The impact of COVID-19 on mothers and young children is substantial (Zhu et al., 2020), with many new precautions being implemented in labour and delivery units at hospitals. Many organizations, such as the Centers for Disease Control and Prevention (CDC) (2020), recommend that mothers with young children take the proper precautions to reduce their risk of getting sick, as this protects both themselves and their children. These precautions include frequent hand hygiene, frequent disinfection of surfaces and objects, and covering their mouth if they cough or sneeze (CDC, 2020; Davanzo, 2020; World Health Organization (WHO), 2020a, b).

During this crucial period, there have been discrepancies in the initially available literature about the recommendation of short-term separation of COVID-19 positive mothers from their infant following childbirth, direct breastfeeding, and use of expressed breastmilk (Davanzo, 2020; Favre et al., $2020 \& \mathrm{Li}$ et al., 2020). The World Health Organization (WHO 2020a, c) recommends breastfeeding mothers continue to breastfeed during the COVID-19 pandemic as the benefits outweigh the risks. Breastfeeding is well known to have immunological benefits, such as decreasing rates of infant morbidity and mortality, with links to lower respiratory infection rates and diarrhea (Victora et al., 2016). Also, longer breastfeeding duration has shown some associations with a more secure mother-baby attachment (Tharner et al., 2012). Breastfeeding is recognized as providing nutritional, developmental, psychological, environmental, and economic benefits that are essential to save the lives of young children, including "at-risk" children (WHO, 2020c). In a retrospective case study of 22 mothers who tested positive for COVID-19 before giving birth, $90.9 \%(n=20)$ breastfed their infants, with no newborns contracting COVID-19 (Pereira et al., 2020). According to the results of their study, breastfeeding is safe during COVID-19 with the proper precautions. WHO strongly recommends breastfeeding of infants and children under the age of 2 years to promote all aspects of their health, growth, and development during difficult circumstances such as pandemic (WHO, 2020c). This suggested the crucial need to promote, protect, and support breastfeeding practices during the COVID-19 pandemic by designing a knowledge dissemination tool that is based on evidence, easy to understand, accessible, user-friendly, and relevant.

On January 25, 2020, Canada confirmed its first case of COVID-19 linked to travel from the epicentre of the virus, Wuhan, China (Government of Canada, 2020). In Canada, as of May 19, 2020, women accounted for $55 \%$ of the reported COVID-19 cases, and 27\% of cases overall fall into the age range of 20 to 39 years (Public Health Agency of Canada, 2020). According to Statistics Canada (2018), Canadian women are increasingly giving birth to their first child at an older age, with the average age of women giving birth being 30.2 years old in 2010. A recent table (Statistics Canada, 2021) shows that in 2019, mothers giving birth between the ages of 30 and 34 accounted for $36.3 \%$ of births in Canada; those between 35 and 39, for 19.8\%; and those between 40 and 44, for just over 4\%. During this COVID-19 pandemic, many breastfeeding mothers in Canada are unable to have regular contact with healthcare professionals to avail themselves of breastfeeding counselling. Moreover, the technical language of the breastfeeding guidelines and discrepancies in the initially available literature on breastfeeding led to various misconceptions around breastfeeding during the pandemic. The 
lack of easy-to-understand, evidence-based, and accessible resources available on breastfeeding during COVID-19 suggested the crucial need to promote, protect, and support breastfeeding during COVID-19 through a knowledge mobilization project targeting Canadian mothers, the general public, and organizations providing direct and indirect care/ services to mothers with young children, including vulnerable groups of Indigenous, refugee, and immigrant mothers.

\section{Objectives}

The goal of this knowledge mobilization project was to develop and disseminate an evidence-based, easy-to-understand, accessible, user-friendly, and relevant knowledge mobilization tool, i.e., an animated video on "Breastfeeding during COVID-19", to breastfeeding mothers in Canada who may not have access to breastfeeding services during the pandemic. Furthermore, the project intended to address misconceptions around breastfeeding during COVID-19 and raise public awareness on safe infant feeding practices during this pandemic. Additionally, the goal was to create a useful resource for the health sectors, post-secondary institutes, and organizations offering direct or indirect care to breastfeeding mothers in Saskatchewan and Canada.

\section{Intervention}

This knowledge mobilization project was undertaken in four phases.

\section{Phase 1: Needs assessment and collaboration}

As an initial step, informal discussions were held with the breastfeeding mothers, service providers, and community partner ${ }^{1}$ in identifying the gaps with respect to lactation counselling facilities during the COVID-19 pandemic. In collaboration with the community partner, breastfeeding mothers were involved in framing the project objectives, conceptualizing the e-resource, and offering feedback on the usefulness of the e-resource during COVID-19.

\footnotetext{
${ }^{1}$ Community parter refers to a community-based organization that provides services to the community for a specific cause. The community partner in this knowledge mobilization project was United Way Regina and was involved in knowledge dissemination to the stakeholders and end-users. United Way Regina works with a multitude of organizations across sectors with the aims of improving lives of people and building community by engaging individuals and mobilizing collective actions.
}

\section{Phase 2: Review of evidence}

As a next step, breastfeeding guidelines and position statements on promotion, protection, and support of breastfeeding during COVID-19 released by the public health agencies, international breastfeeding organizations, or agencies recognized by the United Nations were reviewed and analyzed. In collecting information on breastfeeding recommendations during the COVID-19 pandemic, literature was gathered by manually searching Google using phrases such as "COVID19 and breastfeeding," "SARS-CoV-2 and breastfeeding," and "coronavirus and breastfeeding" and various derivatives. Also, resources were collected through a manual review of trusted health organizations' resources provided in their recommendations. Resources were included if they provided information regarding recommendations for mothers who are breastfeeding during the COVID-19 pandemic, whether they were confirmed to have COVID-19 or not. Organizations were excluded if they were not reputable (i.e., if not a public health agency, international breastfeeding organization, or agency recognized by the United Nations), if they contained commercial advertising, or if they did not contain information relevant to breastfeeding mothers and COVID-19. A total of 23 organizations' recommendations were included in an information table (Table 1), some of which had multiple statements or original resources that were included in the table. The search involved organizations at the local, national, and international levels. These organizations included the World Health Organization, UNICEF, Centers for Disease Control and Prevention (CDC), Canadian Paediatric Society, Royal College of Obstetricians \& Gynecologists, Public Health Agency of Canada, Saskatchewan Health Authority, International Lactation Consultant Association (ILCA), and the Government of Canada. The date range of the resources included in the table was March 5 to May 7, 2020. The search was concluded when recommendations became repetitive. Evidences gathered from breastfeeding guidelines and position statements were initially organized (see Table 1). As a next step, the gathered information was analyzed manually to derive recommendations for breastfeeding mothers during the COVID-19 pandemic (see Table 2).

\section{Phase 3: E-resource creation}

Given the evidence from verifiable and reliable sources, and gap analysis undertaken with the patients, service providers, and community partner, an animated video for the breastfeeding mothers was conceptualized and developed (https://youtu.be/rbsK ypeOO4). To formulate this 5-minute animated video, a professionally trained animated video specialist cum video editor was hired. The video covered content on the importance of breastfeeding during the COVID-19 pandemic, ways breastfeeding mothers can protect themselves 
Table 1 COVID-19 breastfeeding recommendations

Organization Organization name Recommendations if positive/suspected Recommendations if negative

no.

World Health Organization (2020, April • "The COVID-19 virus has not, to date, 28)

Frequently Asked Questions: Breastfeeding and COVID-19 for health care workers been detected in the breastmilk of any mother with confirmed/suspected that COVID-19 would be transmitted COVID-19. It appears unlikely, therefore, through breastfeeding or by giving breastmilk that has been expressed by a mother who is confirmed/suspected to have COVID-19" (WHO, 2020e, para. 1)

- Skin to skin contact and breastfeeding immediately after birth is recommended even if the mother is suspected to be COVID-19 positive, as the benefits are greater than the potential risks of COVID-19 transmission (WHO, 2020e)

- The continuation of breastfeeding is recommended if the mother tests positive for COVID-19, while also implementing proper hygiene practices (WHO, 2020e)

- Hygiene practices when positive for COVID-19 and breastfeeding include washing hands with soap and water or an alcohol-based hand rub frequently and "wearing a medical mask if available, to reduce the possibility of droplets with COVID-19" (WHO, 2020e, para. 5) spreading to the infant while feeding

- Handwashing should be done before touching an infant (WHO, 2020e)

- In the event that you cough or sneeze, do so into a tissue and immediately wash hands with soap and water or an alcohol-based hand rub (WHO, 2020e)

- Surfaces should be regularly cleaned and disinfected, especially if a mother is confirmed/suspected to have COVID-19

- A breastfeeding mother with confirmed/suspected COVID-19 without access to a medical mask should still breastfeed, while also maintaining other hygiene precautions (WHO, 2020e)

- "If a mother confirmed/suspected to have COVID-19 has just coughed over her exposed breast or chest, then she should gently wash the breast with soap and warm water for at least 20 seconds prior to feeding" (WHO, 2020e, para. 14), however, it is not necessary to wash the breast before every infant feeding (WHO, 2020e)

- If a mother with suspected or confirmed COVID-19 is unable to breastfeed, expressed breast milk is the best alternative method of infant feeding and is primarily done with hand expression or with the use of a mechanical pump (WHO, 2020e). Hands should be washed and the mechanical equipment cleaned properly prior to use (WHO, 2020e)

- A mother who was unable to breastfeed due to illness caused by COVID-19 and has
- In cases where COVID-19 is present in a mother's community, breastfeeding is still recommended, as it is what is healthiest for the infant and there is no evidence that COVID-19 can be transmitted through breast milk (WHO, 2020e)

- Despite a lack of risk of having COVID-19, if a mother is using a breast pump or milk storage containers, the equipment should be cleaned after each use with soap and water or in the dishwasher if possible (WHO, 2020e) 
Table 1 (continued)

Organization Organization name Recommendations if positive/suspected Recommendations if negative

no.

UNICEF (2020, April 16)

Navigating pregnancy during the coronavirus disease (COVID-19)
UNICEF: (n.d.)

Coronavirus disease (COVID-19): What parents should know
UNICEF, Global Technical Assistance Mechanism for Nutrition, \& Global Nutrition Cluster (2020, March 30) Infant \& young child feeding in the context of Covid-19 recovered may begin breastfeeding as soon as she feels well enough (WHO, 2020e)

- Newborns and infants are considered to be at low risk of COVID-19 infection, and of the cases of COVID-19 in young children, most experienced mild symptoms or were asymptomatic (WHO, 2020e)

- For women with confirmed or suspected COVID-19, infant formula is not safer than breastmilk, and actually has more associated risks (WHO, 2020e)

- All research shows that COVID-19 is not present in breastmilk and cannot be transmitted to an infant through breastmilk (UNICEF, 2020)

- Mothers who believe they have COVID-19 should seek medical attention and follow the medical advice of their healthcare provider (UNICEF, 2020)

- Precautions for COVID-19 positive mothers who are breastfeeding include wearing a facemask around their infant, ensuring proper hand hygiene before and after handling the infant, and disinfecting surfaces they come into contact with (UNICEF, 2020)

- If a mother is too ill to breastfeed, she should feed her infant with expressed breastmilk via a cup and/or spoon that is clean (UNICEF, 2020)

- Mothers with COVID-19 symptoms such as fever, cough, or difficulty breathing should seek medical attention early and follow the advice of medical professionals (UNICEF, 2020)

- Mothers who test positive for COVID-19 should continue to breastfeed (UNICEF, 2020)

- Precautions for COVID-19 positive mothers who are breastfeeding include wearing a facemask around their infant, ensuring proper hand hygiene before and after handling the infant, and disinfecting surfaces they come into contact with (UNICEF, 2020)

- Mothers who are confirmed or suspected to have COVID-19 should use the recommended hygiene precautions when feeding at home and should continue to breastfeed their infant or newborn (UNICEF Global Nutrition Cluster, 2020)

- It is safe to breastfeed and breastfeeding is encouraged if their infant is suspected or confirmed to have COVID-19 or any other illness (UNICEF Global Nutrition Cluster, 2020)

- Skin to skin contact with breastfeeding is recommended despite the mother or infant having a suspected or confirmed COVID-19 diagnosis (UNICEF Global Nutrition Cluster, 2020) 
Table 1 (continued)

Organization Organization name Recommendations if positive/suspected Recommendations if negative

no.

Canadian Paediatric Society (2020, April 6)

Breastfeeding when mothers have suspected or proven COVID-19
- If the mother is COVID-19 positive, probable, or suspected to be positive, rooming-in practices immediately following birth are still recommended as they are important for establishing breastfeeding

(UNICEF Global Nutrition Cluster, 2020)

- A mother displaying respiratory symptoms should implement the following practices: wear a face mask near the infant, wash hands before and after having contact with the child, and clean/disinfect routinely touched surfaces (UNICEF Global Nutrition Cluster, 2020)

- If the mother is expressing milk, she should wash her hands before touching her breast manually or touching any of the breast pumping equipment (UNICEF Global Nutrition Cluster, 2020)

- A clean cup or spoon should be used to feed the child expressed breastmilk (UNICEF Global Nutrition Cluster, 2020)

- For women even with severe cases of COVID-19, breastfeeding can continue (International Lactation Consultant Association, 2020)

- A breastfeeding mother who is symptomatic should take every precaution in order to avoid spreading the virus to her baby, and these precautions include wearing a face mask when near the infant, cleaning/disinfecting her hands and breast prior to coming into contact with the infant, and proper sanitization of the bottle and pump parts if breast milk expression is being used (American College of Obstetricians and Gynecologists, 2020)

- The American College of Obstetricians and Gynecologists (2020) recommends having someone who is healthy breastfeed the infant if the mother is COVID-19 positive, if possible

- Though it has not yet been found in breastmilk, we cannot yet be certain that COVID-19 cannot be transmitted through breastmilk (American College of Obstetricians and Gynecologists, 2020)

- Mothers with respiratory symptoms should don a surgical mask when they are near their infant, as well as perform hand hygiene prior to handling the infant (Canadian Paediatric Society, 2020)

- If COVID-19 positive, the mother should use soap and water to clean the breast thoroughly before initiating breastfeeding (Canadian Paediatric Society, 2020). If the mother is pumping breast milk, she should thoroughly clean all of the necessary equipment before feeding expressed milk to her infant (Canadian Paediatric Society, 2020)
- In cases of COVID-19, breastfeeding should continue, as is supported by international health guidelines (International Lactation Consultant Association, 2020)

- The decision about whether or not to breastfeed in the midst of the COVID-19 pandemic remains a choice determined by the mother, family, and healthcare professions (American College of Obstetricians and Gynecologists, 2020)

- Right now, though there is no evidence the virus can be spread through breast milk, the concern is that a mother will transmit the virus to the infant through respiratory droplets while breastfeeding (American College of Obstetricians and Gynecologists, 2020)

- People symptomatic with COVID-19 should not visit the mother and her baby (Canadian Paediatric Society, 2020) 
Table 1 (continued)

Organization Organization name

no.
Recommendations if positive/suspected
- Household surfaces should be cleaned frequently if the mother comes into contact with them regularly (Canadian Paediatric Society, 2020)

- Mothers who are too ill to breastfeed should be encouraged to express breastmilk for infants, and it is safe to do so (Canadian Paediatric Society, 2020)

- COVID-19 has yet to be detected in breastmilk; however, we cannot be certain that the virus cannot be spread through breastmilk (CDC, 2020)

- Breastmilk benefits infants' nutrition and protection against diseases (CDC, 2020)

- A facemask should be worn and hand hygiene should be performed if the mother is sick (CDC, 2020)

- Mothers who are sick and choose to express breastmilk should use a breast pump and wash their hands and the pump and other equipment before feeding infants $(\mathrm{CDC}$, 2020). All breast pumping equipment should be properly cleaned following each use (CDC, 2020)

- According to the CDC (2020), “...consider having someone who is well feed the expressed breast milk to the infant" (p. 2)

Centers for Disease Control and Prevention (2020, April 6) Considerations for inpatient obstetric healthcare settings
Dietitians of Canada (2020, April 8) Advice for the general public about COVID-19
- Mothers who make the choice to continue to breastfeed should wear a face mask and wash their hands prior to feeding their infant at home and if in hospital (CDC, 2020)

- In the hospital setting, in the case that the infant and mother are temporarily separated, the mother should be encouraged to manually express breastmilk to establish a milk supply or a breast pump should be used (CDC, 2020)

- To protect the infant, everything that comes into contact with the breastmilk should be cleaned and disinfected thoroughly (CDC, 2020)

- Handwashing should be done before breastfeeding the infant, and the mother should wear a mask (Dieticians of Canada, 2020; Society of Obstetricians and Gynaecologists of Canada, 2020)

- Transmission of antibodies to infants through breast milk is possible, though there is minimal evidence of transmission (Dieticians of Canada, 2020; Society of Obstetricians and Gynaecologists of Canada, 2020).

- It is recommended that hand hygiene be performed before touching the infant or other breastfeeding equipment, coughing and sneezing be avoided around the infant, a face mask be worn if it is available when breastfeeding, and the breast pump if one is being used be cleaned properly after each use (Dieticians of Canada, 2020)
Recommendations if negative 
Table 1 (continued)

Organization Organization name Recommendations if positive/suspected Recommendations if negative

no.

Government of Canada (2020, April 30)

Pregnancy, childbirth and caring for newborns: Advice for mothers during COVID-19

Academy of Breastfeeding Medicine (2020, March 10)

ABM statement on coronavirus 2019

(COVID-19)
- It is recommended that when expressing breastmilk, guidelines on how to sterilize the equipment be followed (Dieticians of Canada, 2020)

- When in contact with her baby, a mother should avoid coughing or sneezing and hand hygiene should be performed before and after handling the baby (Government of Canada, 2020)

- Breastfeeding has an insignificant role in the transmission of other respiratory diseases and should be continued in the midst of COVID-19 (Government of Canada, 2020)

- In the case of a COVID-19 positive mother, a mask should always be worn while breastfeeding and if one is not available, she should cover the infant with a towel or blanket (Government of Canada, 2020)

- A COVID-19 positive mother can stay in the same room as her infant, particularly to initiate bonding with the infant when breastfeeding is being established (Government of Canada, 2020)

- Breast pump equipment should be properly cleaned/sterilized between uses (Government of Canada, 2020)

- If the mother is feeling too ill to breastfeed, a healthy individual should be asked to feed and care for the baby, and expressed milk or formula should be used to feed the infant (Government of Canada, 2020)

- It is not known for certain if mothers with COVID-19 can transmit the virus to their infant through breast milk (Academy of Breastfeeding Medicine, 2020)

- "A mother with confirmed COVID-19 or who is a symptomatic person-under-investigation (PUI) for COVID-19 should take all possible precautions to avoid spreading the virus to her infant, including washing her hands before touching the infant, and wearing a face mask, if possible, while feeding at the breast (Academy of Breastfeeding Medicine, 2020, para. 5)

- "If expressing breast milk with a manual or electric breast pump, the mother should wash her hands before touching any pump or bottle parts and follow recommendations for proper pump cleaning after each use. If possible, consider having someone who is well care for and feed the expressed breast milk to the infant" (Academy of Breastfeeding Medicine, 2020, para. 5)

- Hand hygiene includes using alcohol-based hand rub or washing hands with soap and water for at least 20 seconds (Academy of Breastfeeding Medicine, 2020)
- When physical distancing cannot be ensured, ask other household members to wear a mask as well (Government of Canada, 2020).

- Sharing of breast pump equipment or bottles is not recommended (Government of Canada, 2020).

- When in hospital, breastfeeding is the decision of the mother and the family (Academy of Breastfeeding Medicine, 2020) 
Table 1 (continued)

Organization Organization name

no.
Recommendations if positive/suspected

Recommendations if negative
- "Persons with confirmed COVID-19 infection should remain separate (home isolation precautions) from other family members and friends or neighbors including the infant, except for breastfeeding. Ideally there is another uninfected adult to care for the infant's needs including feeding the infant expressed breastmilk if the mother is expressing milk and working to maintain her supply of milk. The mother should practice careful handwashing and use of a mask as noted above for at least 5-7 days until cough and respiratory secretions are dramatically improved" (Academy of Breastfeeding Medicine, 2020, para. 7)

- When in hospital, breastfeeding is the decision of the mother and the family (Academy of Breastfeeding Medicine, 2020)

- While in hospital, if the mother is COVID-19 positive, temporarily separating the mother and infant may be necessary if the mother needs medical attention, while encouraging the mother to express breastmilk if she intends to breastfeed (Academy of Breastfeeding Medicine, 2020)

- If expressing breastmilk, hand washing should be done prior to doing so and breastfeeding equipment should be properly cleaned (Academy of Breastfeeding Medicine, 2020)

- Rooming-in can be done in a hospital if the proper precautions of handwashing and wearing a face mask are maintained (Academy of Breastfeeding Medicine, 2020)

- Breastmilk that is expressed by a mother who is requiring medical attention may be fed to the infant by a healthy individual (Academy of Breastfeeding Medicine, 2020)

- "LLLI supports the World Health Organization's (WHO) recommendation for breastfeeding immediately after birth even when a positive COVID-19 test has been obtained" (LLLI, 2020, para. 3)

- Breastfeeding is an important means of protection for any infant born to a COVID-19 positive mother or a close family member who is COVID-19 positive (LLLI, 2020)

- If a mother becomes ill, it is imperative that breastfeeding is continued whenever possible, given the immunological benefits that breastmilk provides infants (LLLI, 2020)

- If the mother becomes too ill, expressing or pumping breast milk is the next best
- Breastfeeding protects infants from illness and can reduce the severity of illness (LLLI, 2020) 
Table 1 (continued)

Organization Organization name Recommendations if positive/suspected Recommendations if negative

no.

11

Royal College of Obstetricians \&

Gynaecologists (2020, April 17)

Coronavirus (COVID-19) infection in pregnancy: Information for healthcare professionals

UN Population Fund (UNFPA) (2020, March 5)

UNFPA statement on novel coronavirus (COVID-19) and pregnancy

BC Centre for Disease Control (2020, April 17)

Interim guidance: Public health management of cases and contacts associated with novel coronavirus (COVID-19) in the community

Society of Obstetricians and Gynaecologists of Canada (2020, March 13)

Updated SOGC Committee opinion COVID-19 in pregnancy

University of Saskatchewan (2020, May 1)

COVID-19 FAQS option, and if the mother is too ill to do so, donor milk is recommended (LLLI, 2020)

- "The main risk of breastfeeding is the close contact between the baby and the woman, who is likely to share infective droplets" (Royal College of Obstetricians \& Gynaecologists, 2020, p. 34)

- To reduce the spread of the virus to an infant, consider having someone who is healthy feed the infant; further, wash hands before making contact with the baby, bottles, or breast pump (Royal College of Obstetricians \& Gynaecologists, 2020)

- In addition, avoid coughing or sneezing on the baby, and consider wearing a mask when around the infant (Royal College of Obstetricians \& Gynaecologists, 2020)

- If pumping, follow recommendations outlining how to properly clean the pump equipment after each use (Royal College of Obstetricians \& Gynaecologists, 2020)

- Women breastfeeding should not be separated from their infants, due to the lack of evidence that respiratory viruses can be spread to newborns via breastmilk (UNFPA, 2020; UNICEF, 2020)

- Breastfeeding can be continued as long as the mother is feeling healthy enough to do so (UNFPA, 2020). If the mother is healthy enough to breastfeed, she should wear a mask anytime she is near the infant, and she should disinfect surfaces that may be contaminated (UNFPA, 2020)

- A mother who is too ill to continue breastfeeding should express milk for the infant using a clean cup or spoon, wear a mask and perform hand hygiene (UNFPA, 2020)

- As supported by the World Health Organization, if a mother is COVID-19 positive, she should wear a non-medical mask when she is near the infant or breastfeeding, as well as perform hand washing before and after close contact (BC Centre for Disease Control, 2020)

- Women should wash their hands and wear a mask while breastfeeding (Society of Obstetricians and Gynaecologists of Canada, 2020)

- There is limited evidence, but antibodies may be transmitted to the infant through breast milk (Society of Obstetricians and Gynaecologists of Canada, 2020)

- Mothers who are SARS-CoV-2 positive and are still breastfeeding should use the following precautions: wear a mask, perform hand hygiene before touching the infant, disinfect frequently touched surfaces in your home, and clean the breast with soap and water before breastfeeding (University of Saskatchewan, 2020)
- Breastfeeding mothers can continue to breastfeed, as it poses a low risk to the infant as opposed to the many benefits of breastfeeding (BC Centre for Disease Control, 2020) 
Table 1 (continued)

Organization Organization name Recommendations if positive/suspected Recommendations if negative

no.

American Academy of Family

Physicians (2020, April 15)

$A A F P$ statement on breastfeeding and COVID-19
New Zealand Ministry of Health (2020, May 1)

Breastfeeding advice for women who have a confirmed or probable case of COVID-19
- If expressing milk, perform hand hygiene, and clean breast pump equipment before and after each use (University of Saskatchewan, 2020)

- If the mother is too ill to breastfeed, expressed milk is safe to feed to the infant (University of Saskatchewan, 2020)

- "The American Academy of Family Physicians (AAFP) recommends breastfeeding for the majority of infants except in the case of certain medical conditions. Limited evidence suggests that SARS-CoV-2, the virus that causes COVID-19, is spread via respiratory droplets, and to date, studies have not detected SARS-CoV-2 and similar coronavirus infection in breast milk. Therefore, the AAFP recommends promotion of breastfeeding and parent-infant bonding, and avoidance of parent-infant separation whenever possible" (American Academy of Family Physicians, 2020, para. 1)

- "If a parent has been diagnosed with COVID-19 or was exposed and has symptoms, it is still reasonable to breastfeed and/or provide expressed milk for the infant. In addition to masks and handwashing, extra mechanisms to reduce exposure are suggested such as limiting contact with the infant outside of breastfeeding and the use of expressed breast milk fed to the infant by another household member who does not have COVID-19. If expressing breast milk with a manual or electric pump, the parent should wash their hands before touching any pump or bottle parts and sanitize the pump after each use" (AAFP, 2020, para. 3)

- If a mother is COVID-19 positive or suspected to be positive for COVID-19, breastfeeding is still a choice with the implementation of precautions (New Zealand Ministry of Health, 2020)

- A surgical mask should be worn to reduce the risk of spreading the virus to your infant (New Zealand Ministry of Health, 2020)

- Hand hygiene should be performed thoroughly before feeding the infant, and the touching or kissing of the infant's face should be avoided (New Zealand Ministry of Health, 2020)

- If you are not well enough to breastfeed, expressed breast milk should be fed to the baby via a bottle using the above precautions (New Zealand Ministry of Health, 2020)

- Breastfeeding does not need to be interrupted in the event that you become ill with COVID-19 in the days or weeks
- "If a parent has been exposed to COVID-19 but is asymptomatic, breastfeeding is a reasonable choice" (AAFP, 2020, para. 2)

- Considering the known benefits of breastfeeding and the fact that there is no evidence of COVID-19 being transmitted through breastmilk, breastfeeding should continue throughout the COVID-19 pandemic (New Zealand Ministry of Health, 2020) 
Table 1 (continued)

Organization Organization name Recommendations if positive/suspected Recommendations if negative

no.

18

Alberta Health Services (2020, May 7)

COVID-19 and pregnancy, birth, postpartum, and breastfeeding: Information for expectant and new parents

Better Outcomes Registry \& Network Ontario (BORN Ontario) (2020, May 7)

COVID-19 infection and pregnancy following birth (New Zealand Ministry of Health, 2020)

- Currently, there is no evidence to suggest that COVID-19 can be passed on to your baby through breastmilk, and therefore breastfeeding is recommended even if you are COVID-19 positive (Alberta Health Services, 2020)

- Because COVID-19 is spread through respiratory droplets such as through coughing and sneezing, or touching contaminated objects, perform hand hygiene before and after touching your baby and wear a face mask when possible when near your baby or when you are breastfeeding; in addition, it is important to clean your chest before you breastfeed your infant (Alberta Health Services, 2020)

- If you are using a breast pump, perform hand hygiene before touching the breast pump and other breastfeeding equipment; in addition, clean the equipment after each use (Alberta Health Services, 2020)

- If a face mask is unavailable, use multiple layers of cloth, such as a scarf or bandana to cover your nose and mouth while you breastfeed your baby (Alberta Health Services, 2020)

- Perform hand hygiene before applying the mask to your face and wash the mask after each use; note that face masks are more effective than alternative masks (Alberta Health Services, 2020)

- If the mother is unwell, have someone else who is healthy feed the baby via a bottle (Alberta Health Services, 2020)

- Decisions about whether the mother wants to breastfeed or not should be discussed with the family on an individualized case-by-case basis (Better Outcomes Registry \& Network Ontario, 2020)

- "Breastfeeding is still supported as the virus has not been found in breastmilk. However, mothers with confirmed or suspected COVID-19 should wear a facemask while breastfeeding or holding their baby skin-to-skin, and wash their hands before touching the baby, the breast pumps, or the bottles. The breast area should also be washed with soap and water before breastfeeding" (Better Outcomes Registry \& Network Ontario, 2020, para. 14)

- "There is very little evidence at this moment on whether specific protection for COVID-19 can be passed on to the baby through breastfeeding" (Better Outcomes Registry \& Network Ontario, 2020, para. 15)

- Despite the COVID-19 pandemic, breastfeeding is still recommended, as 
Table 1 (continued)

Organization Organization name Recommendations if positive/suspected Recommendations if negative

no.

COVID-19 information for patients: Frequently asked questions for $B C$ Women's patients about novel coronavirus COVID-19
- It is safe to breastfeed, despite having COVID-19 symptoms (BC Women's Hospital, 2020)

- Wear a mask when you are near your baby and wash your hands before touching your baby (BC Women's Hospital, 2020)
21
Saskatchewan Health Authority (SHA) (2020, March 15)

Postpartum and newborn care standard for COVID-19;

(2020, March 15)

Infant feeding, including breastfeeding expressed breast milk, when mother positive, under investigation, or symptomatic for COVID-19
Government of Quebec (2020, April 28) during the coronavirus disease (COVID-19) pandemic

Association of Ontario Midwives (2020, April 7)

Chest/breastfeeding
- "Women may still be accompanied by a support person for their delivery and practice skin-to-skin contact, breastfeeding and rooming-in if the mother and newborn do not require additional care" (Government of Quebec, 2020, para. 11)

- Mothers who are too ill to breastfeed should be encouraged to feed their infant expressed milk, while using the proper precautions (e.g., handwashing and wearing a face mask, if available) to decrease transmission risk to infants (Association of Ontario Midwives, 2020)

- If available, have someone who is feeling well feed the infant the expressed breastmilk if the mother is too ill to do so (Association of Ontario Midwives, 2020) human milk contains antibodies that can help support infants' immune systems (BC Women's Hospital, 2020)

- Wash your hands before and after touching your infants, and wear a mask if you feel unwell (BC Women's Hospital, 2020)

- If you are temporarily separated from your infant, expressing breast milk is encouraged (BC Women's Hospital, 2020)

- It is safe to reuse your breast pump equipment, as long as it is properly cleaned in between uses (BC Women's Hospital, 2020)

"To promote breastfeeding, the usual practices such as skin-to-skin contact, breastfeeding and rooming in with the baby are still encouraged" (Government of Quebec, 2020, para. 9)

- Mothers who decide to breastfeed should perform hand hygiene frequently, particularly before and after handling their infant (Association of Ontario Midwives, 2020)

- Mothers should clean their chest with soap and water before breastfeeding their infant (Association of Ontario Midwives, 2020)

- Mothers should wear a facemask while holding their infant if they have access to one, and avoid coughing or sneezing on their infant (Association of Ontario Midwives, 2020)

- Breast pumping equipment should be thoroughly sterilized prior to use (Association of Ontario Midwives, 2020)

- Frequently touched surfaces should be disinfected regularly (Association of Ontario Midwives, 2020)

- Midwives should encourage mothers to breastfeed while informing the parents about the risks and benefits of breastfeeding for the infant and mother (Association of Ontario Midwives, 2020)
- Close prolonged contact including breastfeeding and skin to skin contact is approved by the Saskatchewan Health Authority (SHA, 2020)

- Women with confirmed or suspected COVID-19 may choose to breastfeed (SHA, 2020)

- The concern remains that COVID-19 could be spread to infants during breastfeeding and feeding with expressed breast milk (SHA, 2020)

- All women with suspected or confirmed COVID-19 should do the following before breastfeeding: perform hand hygiene, wear a face mask (SHA, 2020)

- If using a breast pump, mothers with suspected or confirmed COVID-19 should 
Table 1 (continued)

Organization Organization name Recommendations if positive/suspected Recommendations if negative

no.

wash their hands or use hand sanitizer be-

fore touching breastfeeding equipment, as

well as wash any breastfeeding equipment

between uses (SHA, 2020)

- If using expressed breastmilk, consider

having the infant fed by a person who is

not symptomatic of COVID-19 or

suspected to be positive (SHA, 2020)

- Mothers who plan on breastfeeding and are

temporarily separated from their infant

should be encouraged to express breast

milk in order to establish a milk supply

(SHA, 2020)

and young children from COVID-19, and handwashing technique. Furthermore, it provided information on the preparation of home-made masks, maintaining bonding with newborn/infant during the period of social isolation, assuring good mental health during the pandemic, and using alternate safe feeding options if breastfeeding is not possible. Although the medium of the developed e-resource was English, the subtitles included in the animated video can be translated into many different languages. This feature allowed mothers from diverse cultural groups and language skills to access the e-resource in their preferred language.

\section{Phase 4: Dissemination of e-resource}

The e-resource was disseminated with the support of the community-based partner, which works with a multitude of organizations across public and private sectors in Canada. The "Breastfeeding during COVID-19: An Information Guide" video was distributed to breastfeeding mothers, the general public, health sectors, 113 agencies in Saskatchewan, and governmental/non-governmental organizations working nationally across Canada. These agencies/organizations, which focus predominantly on family and maternal health, shared the video with individuals who may benefit from the information provided in it. This e-resource was also disseminated through social media, including Twitter, YouTube, LinkedIn, Facebook, and the researchers' professional organization website/social media. The link for the animated video on "Breastfeeding during COVID-19" was also distributed to a range of knowledge users, including lactation consultants working across Canada, agencies offering services to mothers and people in mothers' social network, post-secondary institutions in Canada offering training to healthcare professionals, and organizations providing direct and indirect care/services to Indigenous, refugee, and immigrant mothers in Canada.
To promote dialogue and note the impact (utilization) of this knowledge mobilization resource, a Facebook page on "Breastfeeding during COVID-19" was launched where the developed animated video and evidence-based e-resources on breastfeeding during the COVID-19 pandemic were posted. The impact of this animated video was monitored through statistics of views and comments posted by the viewers. The impact of this video was further assessed through comments received from the community-based organizations and endusers on the usefulness of the resource.

\section{Outcomes}

During the COVID-19 pandemic, this knowledge mobilization project met the goal of developing and disseminating an e-resource (animated video) on "Breastfeeding during COVID-19" to the end-users, including breastfeeding mothers, the general public, and organizations providing services to mothers with young children. From May 3 to November 30, 2020, the "Breastfeeding during COVID-19: An Information Guide" e-resource had 1290 views on YouTube. The viewers of the video were in the age demographic of 35 to 44 years of age, according to the YouTube video analytics. The viewership was predominantly female, with $73.8 \%$ of the viewers being female and $26.2 \%$ being male. Over half $(59.3 \%)$ of the viewers watched the video from a mobile device, with an additional $37.3 \%$ watching from a computer, $2.9 \%$ viewing from a tablet, and $0.2 \%$ viewing from a television. YouTube video analytics indicated that viewers shared this e-resource through WhatsApp, Facebook, Facebook messenger, and text message. In regards to location, the majority of the people viewed the video in Canada; additional viewers were from the United States, India, Pakistan, and other countries. These additional viewers may be the Canadian mothers who travelled outside Canada during the 
Can J Public Health (2021) 112:599-619

613

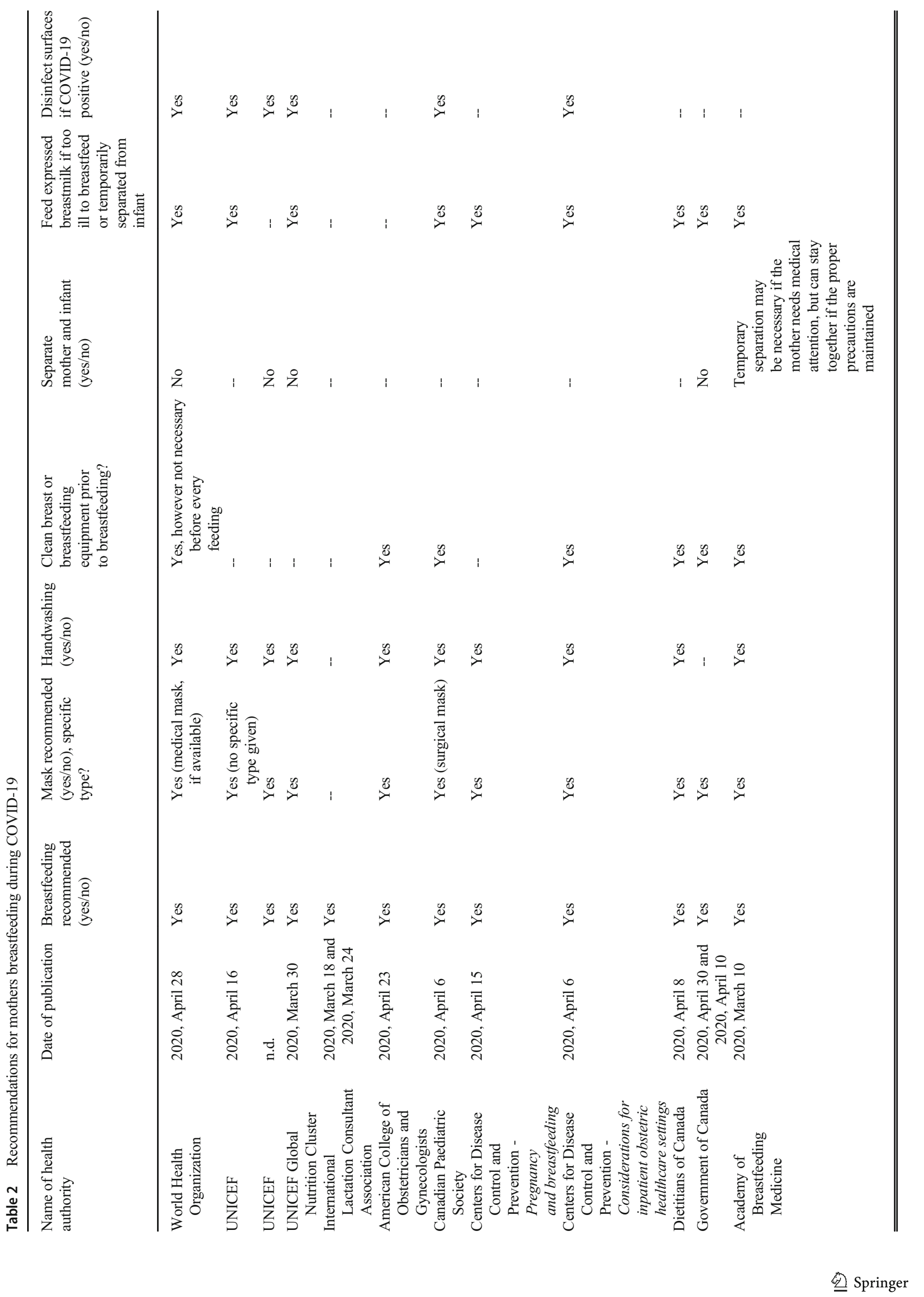




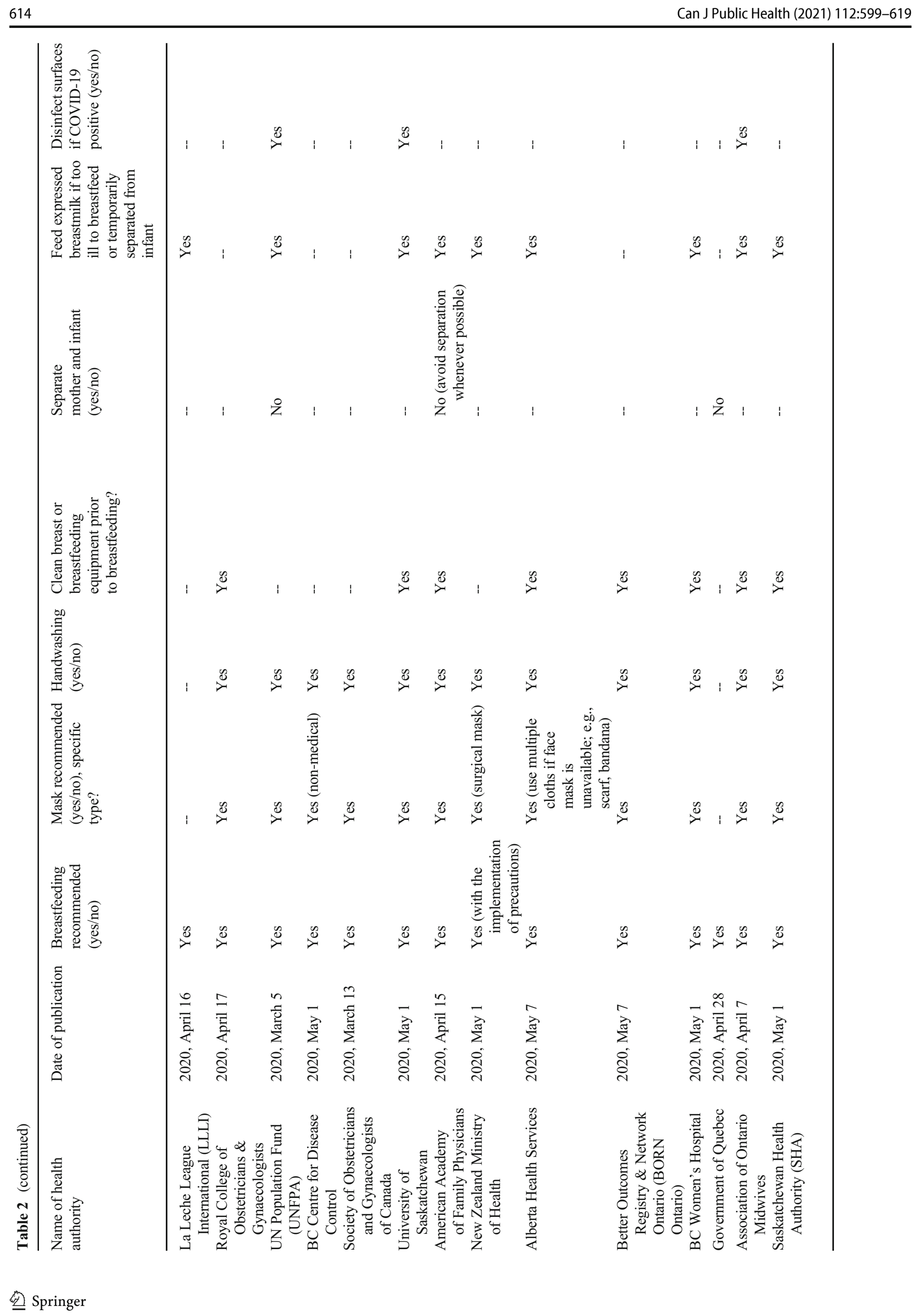


pandemic, people in the social network of the Canadian mothers residing in other countries, practitioners, healthcare providers, service providers, or researchers from outside Canada who wish to adopt this e-resource in their context.

Since launching the "Breastfeeding during COVID-19" Facebook page on May 4, 2020, the breastfeeding support page has gained a total of 236 followers, with 233 people "liking" the page. This page was created to share evidencebased e-resources with mothers who are breastfeeding during the COVID-19 pandemic to promote, protect, and support breastfeeding practices. The "Breastfeeding during COVID19: An Information Guide" YouTube video was shared on this page, along with other evidence-informed resources through mediums such as photos, videos, links, and written posts provided by organizations, including the World Health Organization, UNICEF, CDC, ILCA, and Canadian health authorities. Of the nearly 50 e-resources shared through this Facebook page, the number of people reached ranged from 3 to 3300 people, with engagements ranging from 0 to 122 per post. According to Facebook analytics, the majority of individuals accessed the Facebook page from mobile devices, with a portion using their computer. Overall, the total reach of this Facebook page, defined by the total number of people who have viewed any of the posts on this page, is 3279 people from June 10 to July 6, 2020.

The e-resource was adopted by several organizations, including COVID-19 resource Canada, Health-e-learning: An International Institute of Human Lactation, Saskatchewan Health Authority, Breastfeeding Committee of Saskatchewan, post-secondary institutions in Saskatchewan, and several other health agencies in Canada. Various organizations disseminated the video in their newsletter, an online blog post on their website, and social media (mainly Twitter and Facebook page), which gained many shares.

\section{Video resource viewer feedback}

Analysis of comments posted by the viewers on social media indicated that the developed evidence-based e-resource on "Breastfeeding during COVID-19" was an informative, userfriendly, useful, and easily accessible resource for breastfeeding mothers who were in self-isolation with little access to healthcare services during the pandemic. Knowledge shared on the benefits of breastfeeding during the current COVID-19 global pandemic encouraged breastfeeding mothers to sustain their breastfeeding practices during the pandemic and guided them to adopt necessary precautions to prevent themselves and their young child from getting COVID-19. The developed animated video on "Breastfeeding during COVID-19: An Information Guide" served as a resource for the health sector and organizations offering care to breastfeeding mothers within and outside Canada.
Several strengths of the video resource were identified by viewers. These strengths include its delivery of informative, relevant, and easy-to-understand information, the engaging visual representations of the information being presented, the diversity demonstrated in the video, and the quality of the video. In particular, many community-based organizations providing services to immigrant and refugee mothers in Canada praised the ease with which their clients who are not fluent in English were able to utilize the video resource. Comments directly from YouTube indicated that viewers found the video to be informative, need-based, timely, and helpful for breastfeeding mothers who may have questions concerning breastfeeding during this pandemic.

\section{Discussion}

Breastfeeding young children during the COVID-19 pandemic could save costs during economically challenging times, promote health and prevent illnesses among young children, boost infants' immunity and protect them from infections, enhance mother-child bonding, and promote brain development of young children during the most sensitive period of their lives. During the COVID-19 pandemic, WHO (2020d) fully supports and encourages the continuation of breastfeeding for mothers with newborns and infants up to and beyond 2 years of age. However, a mother displaying symptoms of COVID-19 should take the proper precautions to decrease transmission, including wearing a medical mask when near the child, routinely cleaning and disinfecting, and handwashing (WHO, 2020d).

This knowledge mobilization project was conceptualized to increase awareness among breastfeeding mothers and the general public on safe infant feeding practices during the COVID-19 pandemic, clarifying myths and misconceptions associated with breastfeeding practices, guiding breastfeeding mothers to make informed decisions around child feeding practices, and offering meaningful guidance in simple language through a short online animated video and Facebook page. The developed e-resources served as an avenue to provide accessible and evidence-based information to the breastfeeding mothers in Saskatchewan and Canada during the pandemic who may lack access to information due to self-isolation, social distancing, and avoiding visiting healthcare facilities.

The undertaken project underscored that electronic resources can be valuable tools to promote, protect, and support the breastfeeding practices of mothers. Literature also highlights that mothers should have online resources accessible to facilitate their decision-making around breastfeeding (Radzyminski \& Callister, 2015). Breastfeeding mothers often seek health information during their postpartum period and appreciate health information that is provided online by clinical professionals (Bernhardt \& Felter, 2004). A study explored breastfeeding 
mothers' preference for various technology mediums and identified "breastfeeding" being one of the most regularly accessed subjects using technology, mainly smartphone apps, pregnancy and parenting websites, Internet search engines, email, and online videos (Demirci et al., 2016). Electronic forums and resources are reported to provide need-based advice and emotional support about breastfeeding and parenting (Cowie et al., 2011). A study that implemented a breastfeeding promotion video among a group of pregnant women found that the video promoted breastfeeding and addressed breastfeeding barriers (Khoury et al., 2002). Another study found that an online intervention consisting of two videos and a tip sheet helped mothers cope with challenging breastfeeding experiences and increased breastfeeding satisfaction; in fact, most of the mothers in this study recommended the e-resources to other mothers (Mitchell et al., 2018).

Seeking breastfeeding information from online resources is becoming increasingly popular, especially during the COVID19 pandemic. Literature underscores that mothers may access online breastfeeding resources for a variety of reasons, such as a lack of familial, informational, or professional support. A study conducted in the United Kingdom looked at the active participation of women in an online breastfeeding support group and found the main reasons for accessing the online support group were for informational support, as well as esteem and emotional support (Wagg et al., 2019). Parents may rely more heavily on electronic support groups and online information when face-toface opportunities for interaction are not possible. Online support is always available, whereas healthcare professional support may be limited at times (Regan \& Brown, 2019), especially during a global pandemic. Literature underscores that online information can have an impact on breastfeeding success among women (Newby et al., 2015). In a focus group study exploring the use of digital media by women for pregnancy and breastfeeding, participants reported that information from digital media is practical, immediately available, detailed, and professional (Lupton, 2016). In a study conducted by Regan and Brown (2019), breastfeeding mothers expressed that online breastfeeding support provided reassurance and was more easily accessible than face-to-face interactions, hence contributing to their sustained breastfeeding. Videos through digital platforms such as YouTube were cited as being useful for parents, as the information was available to review repeatedly (Lupton, 2016).

The creation of the e-resources in this project had the key goal to support mothers seeking information on breastfeeding during the COVID-19 pandemic by providing accessible and evidence-based information in one place. Literature underscores that informational websites do not always provide all of the information parents are seeking regarding topics such as breastfeeding, which is why online resources linked to these websites can be useful to support parents (Cowie et al., 2011). Though online forums and social media can be useful in supporting breastfeeding mothers, it is possible that medical advice can be given by individuals who are not trained professionals and this can lead to misinformation (Regan \& Brown, 2019). This indicates the importance of e-resources that are evidence-based.

\section{Recommendations and implications}

The use of e-resources during a pandemic, when mothers do not have access to certain healthcare services, is important to promote, protect, and support breastfeeding. Healthcare providers and nurses should recommend and use evidence-based e-resources to better promote and protect breastfeeding during the COVID-19 pandemic. To meet the needs of the breastfeeding mothers from diverse cultural groups, the eresources recommended should be evidence-based and userfriendly, use simple language, and be easily accessible, and must be available in different languages.

Development of knowledge mobilization resources during the COVID-19 pandemic, such as the informational video created for women breastfeeding, would strongly benefit from extensive collaboration with patients and a community partner. The utilization of evidence-based information in the creation of these e-resources is essential to focus on developing informational resources that are trustworthy and beneficial for the intended audience.

Furthermore, providing the users of e-resources with a way to offer feedback is helpful to determine the usefulness of the e-resources. As is supported by the findings of the dissemination of the e-resource created, social media can be an effective method of distributing information about breastfeeding practices during COVID-19 to the general public, breastfeeding mothers, service providers, and possibly those who may face language barriers. The creation of breastfeeding support groups through online social media platforms would be helpful to allow mothers to engage in discussions and access reliable information in a central location, rather than having to search for it extensively.

\section{Conclusion}

This evidence-based e-resource facilitated addressing misconceptions around breastfeeding during COVID-19 and raising public awareness on safe infant feeding practices during this pandemic. Patient engagement and collaboration with the community partner facilitated identification of practice-related gaps surrounding breastfeeding during the pandemic and conceptualizing an easy-to-understand evidence-based e-resource. The e-resource was disseminated through social media and distributed to breastfeeding mothers, lactation consultants, the general public, service providers, and various organizations in Canada providing 
direct or indirect services to mothers with young children. Overall, the video was described as an informative, userfriendly, useful, and easily accessible resource by breastfeeding mothers who were in self-isolation with little access to healthcare services during the pandemic, and would be a useful resource for healthcare professionals to disseminate to breastfeeding mothers seeking information on breastfeeding during COVID-19.

\section{Implications for policy and practice}

What are the innovations in this policy or program?

- During this COVID-19 pandemic, many breastfeeding mothers in Canada are unable to have regular contact with healthcare professionals to avail themselves of breastfeeding counselling facilities.

- The technical language of the breastfeeding guidelines and discrepancies in the initially available literature on breastfeeding led to various misconceptions around breastfeeding during the pandemic.

- The lack of easy-to-understand, evidence-based, and accessible resources available on breastfeeding during COVID-19 suggested the crucial need to promote, protect, and support breastfeeding during the pandemic. This was addressed through the development and dissemination of an innovative e-resource entitled "Breastfeeding during COVID-19: An Information Guide" that is evidencebased, easy to understand, accessible, and user-friendly.

What are the burning research questions for this innovation?

- Development of innovative knowledge mobilization tools, such as the e-resource created for women breastfeeding during the COVID-19 pandemic, would strongly benefit from extensive collaboration with patients and community partners, utilization of evidence-based information, using social media for dissemination of need-based information, and providing the users of e-resources with a way to offer feedback to determine the usefulness of the e-resources.

- The creation of breastfeeding support groups through online social media platforms would be helpful to allow mothers to engage in discussions and access reliable information in a central location, rather than having to search for it extensively.

Acknowledgements We are grateful to governmental and nongovernmental agencies in Canada for disseminating the developed knowledge mobilization resource to stakeholders and breastfeeding mothers. We are thankful to breastfeeding mothers and service providers for their valuable comments and feedback on the developed e-resource. We are thankful to Karim Ali for his support in video editing.
Author contributions Dr. Shela Hirani (Associate Professor and Lactation Consultant) as a Principal Investigator was responsible for the conception of the project, collaboration with the patient and community-based patient partners, drafting the proposal, development of the e-resource, and writing and reviewing the manuscript at all stages. Megan Pearce contributed to the literature review, analysis of comments, and write-ups for the sections of the manuscript. Megan is a SCPOR trainee and research assistant on this project. Amanda Lanoway as a representative of our community partner, United Way Regina, was involved in the knowledge dissemination to stakeholders and end-users.

Funding We acknowledge the support of the Saskatchewan Health Research Foundation (SHRF) (funding ID: 5330), University of Regina Faculty of Nursing, Canada (funding ID: 253367), and Saskatchewan Centre for Patient-Oriented Research (funding ID: 241346) for funding this knowledge mobilization project.

Availability of data and material We did not collect data from the participants. The weblink of the developed e-resource is shared in the manuscript. Gathered literature is summarized in Tables 1 and 2.

Code availability Not applicable.

\section{Declarations}

Ethics approval Not applicable.

Consent to participate Not applicable.

Consent for publication Not applicable.

Conflict of interest The authors declare no competing interests.

\section{References}

Academy of Breastfeeding Medicine (ABM). (2020). ABM statement on coronavirus 2019 (COVID-19). https://www.bfmed.org/abmstatementcoronavirus. Accessed 10 May 2020.

Acter, T., Uddin, N., Das, J., Akhter, A., Choudhury, T., \& Kim, S. (2020). Evolution of severe acute respiratory syndrome coronavirus 2 (SARS-CoV-2) as coronavirus disease 2019 (COVID-19) pandemic: A global health emergency. Science of the Total Environment, 730, 138996. https://doi.org/10.1016/j.scitotenv. 2020.138996.

Alberta Health Services. (2020). COVID-19 and pregnancy, birth, postpartum, and breastfeeding: Information for expectant and new parents. https://www.albertahealthservices.ca/assets/info/ppih/ifppih-covid-19-prenatal-postnatal.pdf. Accessed 10 May 2020.

American Academy of Family Physicians. (2020). AAFP statement on breastfeeding and COVID-19. https://www.aafp.org/dam/AAFP/ documents/patient care/public health/AAFP-COVIDBreastfeeding-Policy.pdf. Accessed 19 April 2020.

American College of Obstetricians and Gynecologists. (2020). Novel Coronavirus 2019 (COVID-19). https://www.acog.org/clinical/ clinicalguidance/practice-advisory/articles/2020/03/novelcoronavirus-2019. Accessed 27 April 2020.

Association of Ontario Midwives. (2020). COVID-19 clinical FAQ: Chest/breastfeeding. https://www.ontariomidwives.ca/covid-19clinical-faq. Accessed 15 April 2020.

Bernhardt, J., \& Felter, E. (2004). Online pediatric information seeking among mothers of young children: Results from a qualitative study 
using focus groups. Journal of Medical Internet Research, 6(1), E7. https://doi.org/10.2196/jmir.6.1.e7.

Better Outcomes Registry \& Network Ontario. (2020). COVID-19 infection and pregnancy. https://www.bornontario.ca/en/news/covid-19infectionand-pregnancy.aspx. Accessed 10 May 2020.

BC Centre for Disease Control. (2020). Interim guidance: Public health management of cases and contacts associated with novel coronavirus (COVID-19) in the community. http://www.bccdc.ca/ resourcegallery/Documents/Guidelines\%20and\%20Forms/ Guidelines\%20and\%20Manuals/Epid/CD\%20Manual/Chapter\% 201\%20-\%20CDC/2019-nCoV-Interim Guidelines.pdf. Accessed 20 April 2020.

BC Women's Hospital. (2020). COVID-19 information for patients: Frequently asked questions for BC Women's patients about novel coronavirus COVID-19. https://www.bcwomens.ca/health-info/ covid-19-information-for-patients. Accessed 3 May 2020.

Canadian Paediatric Society. (2020). Breastfeeding when mothers have suspected or proven COVID-19. https://www.cps.ca/en/documents/ position/breastfeeding-when-mothers-have-suspected-or-provencovid-19. Accessed 15 April 2020.

Centers for Disease Control and Prevention. (2020). Protect yourself and your family from COVID-19. https://www.cdc.gov/coronavirus/ 2019-ncov/need-extra-precautions/pregnancybreastfeeding.html? CDC_AA_refVal=https $\% 3 \mathrm{~A} \% 2 \mathrm{~F} \% 2 \mathrm{Fwww} . \mathrm{cdc} . \mathrm{gov} \%$ 2 Fcoronavirus $\% 2$ F 2019 -ncov $\% 2$ Fprepare $\% 2$ Fpregnancybreastfeeding.html. Accessed 25 April 2020.

Cowie, G., Hill, S., \& Robinson, P. (2011). Using an online service for breastfeeding support: What mothers want to discuss. Health Promotion Journal of Australia, 22(2), 113-118. https://doi.org/ 10.1071/he11113.

Davanzo, R. (2020). Breast feeding at the time of COVID-19: Do not forget expressed mother's milk, please. Archives of Disease in Childhood. Fetal and Neonatal Edition. https://doi.org/10.1136/ archdischild-2020-319149.

Demirci, J., Cohen, S., Parker, M., Holmes, A., \& Bogen, D. (2016). Access, use, and preferences for technology-based perinatal and breastfeeding support among childbearing women. The Journal of Perinatal Education, 25(1), 29-36. https://doi.org/10.1891/10581243.25.1.29.

Dietitians of Canada. (2020). Advice for the general public about COVID-19. https://www.dietitians.ca/News/2020/Advice-for-thegeneral-publicabout-COVID-19. Accessed 15 April 2020.

Favre, G., Pomar, L., Qi, X., Nielsen-Saines, K., Musso, D., \& Baud, D. (2020). Guidelines for pregnant women with suspected SARS-CoV2 infection. The Lancet Infectious Diseases, 20(6), 652-653. https:// doi.org/10.1016/S1473-3099(20)30157-2.

Government of Canada. (2020). Coronavirus disease (COVID-19): For health professionals. https://www.canada.ca/en/public-health/ services/diseases/2019-novel-coronavirus-infection/healthprofessionals.html. Accessed 29 April 2020.

Government of Quebec. (2020). Pregnancy, delivery and postnatal period during the coronavirus disease (COVID-19) pandemic. https:// www.quebec.ca/en/health/health-issues/a-z/2019-coronavirus/ information-for-pregnant-women-coronavirus-covid-19/. Accessed 29 April 2020.

International Lactation Consultant Association. (2020). ILCA statement on breastfeeding and lactation support during the COVID-19 pandemic. https://lactationmatters.org/2020/03/18/ilca-statementon-breastfeeding-and-lactation-support-during-the-covid-19pandemic/. Accessed 10 April 2020.

Khoury, A., Mitra, A., Hinton, A., Carothers, C., \& Sheil, H. (2002). An innovative video succeeds in addressing barriers to breastfeeding among low-income women. Journal of Human Lactation, 18(2), 125-131. https://doi.org/10.1177/089033440201800203.
La Leche Leage International (LLLI). (2020). Breastfeeding, Childbirth, and COVID-19. https://www.1lli.org/breastfeeding-childbirth-andcovid-19/. Accessed 20 April 2020.

Li, F., Feng, Z., \& Shi, Y. (2020). Proposal for prevention and control of the 2019 novel coronavirus disease in newborn infants. Archives of Disease in Childhood - Fetal and Neonatal Edition, Archives of Disease in Childhood - Fetal and Neonatal Edition, 4 March 2020. https://doi.org/10.1136/archdischild-2020-318996.

Lupton, D. (2016). The use and value of digital media for information about pregnancy and early motherhood: A focus group study. BMC Pregnancy and Childbirth, 16(1). https://doi.org/10.1186/s12884016-0971-3.

Mitchell, A., Whittingham, E., Steindl, K., \& Kirby, S. (2018). Feasibility and acceptability of a brief online self-compassion intervention for mothers of infants. Archives of Women's Mental Health, 21(5), 553561. https://doi.org/10.1007/s00737-018-0829-y.

New Zealand Ministry of Health. (2020). Breastfeeding advice for women who have a confirmed or probable case of COVID-19. https:// www.health.govt.nz/our-work/diseases-and-conditions/covid-19novel-coronavirus/covid-19-information-specific-audiences/covid19-informationparents-during-alert-level-3/breastfeeding-advicewomen-who-have-confirmed-or-probable-case-covid-19? fbclid= Iw AR 30 g UTuQZxtxEhRt9W A 2IhBiN6TE9pKGOFpLh10NBN5hq2OFFBjqXRpX. Accessed 3 May 2020.

Newby, R., Brodribb, W., Ware, R. S., \& Davies, P. S. (2015). Internet use by first-time mothers for infant feeding support. Journal of Human Lactation, 31(3), 416-424. https://doi.org/10.1177/ 0890334415584319 .

Pereira, A., Cruz-Melguizo, S., Adrien, M., Fuentes, L., Marin, E., Forti, A., \& Perez-Medina, T. (2020). Breastfeeding mothers with COVID-19 infection: A case series. International Breastfeeding Journal, 15(1), 69. https://doi.org/10.1186/s13006-020-00314-8.

Public Health Agency of Canada. (2020). COVID-19 daily epidemiology update. https://www.canada.ca/content/dam/phac-aspc/documents/ services/diseases/2019-novel-coronavirus-infection/surv-covid19epi-update-eng.pdf. Accessed 29 April 2020.

Radzyminski, S., \& Callister, L. (2015). Health professionals' attitudes and beliefs about breastfeeding. The Journal of Perinatal Education, 24(2), 102-109. https://doi.org/10.1891/1058-1243.24.2.102.

Regan, S., \& Brown, A. (2019). Experiences of online breastfeeding support: Support and reassurance versus judgement and misinformation. Maternal \& Child Nutrition, 15(4). https://doi.org/10.1111/ mcn. 12874.

Royal College of Obstetricians \& Gynaecologists. (2020). Coronavirus (COVID-19) infection in pregnancy: Information for healthcare professionals. https://www.rcm.org.uk/media/3892/2020-04-17coronavirus-covid-19-infection-in-pregnancy.pdf. Accessed 19 April 2020.

Saskatchewan Health Authority. (2020). Postpartum and newborn care standard for COVID-19. https://www.saskatchewan.ca/search\#q= breastfeeding\%20and\%20covid-19\&sort=relevancy. Accessed 10 April 2020.

Society of Obstetricians and Gynaecologists of Canada. (2020). Updated SOGC Committee opinion-COVID-19 in pregnancy. https://sogc. $\mathrm{org} / \mathrm{en} / \mathrm{content} /$ featured-news/Committee-Opinion-No-400\%E2\% 80\%93COVID-19-in-Pregnancy-updated-May-14-2020.aspx. Accessed 10 April 2020.

Statistics Canada. (2018). Fertility: Fewer children, older moms. https:// www150.statcan.gc.ca/n1/pub/11-630-x/11-630-x2014002-eng. htm. Accessed 29 April 2020.

Statistics Canada. (2021). Table 13-10-0416-01 Live births, by age of mother. https://doi.org/10.25318/1310041601-eng. Accessed 1 March 2021.

Tharner, A., Luijk, M., Raat, H., Ijendoorn, M. H., BakermansKranenburg, M. J., Moll, H. A., Jaddoe, V. W., Hofman, A., Vernhulst, F. C., \& Tiemeier, H. (2012). Breastfeeding and its 
relation to maternal sensitivity and infant attachment. Journal of Developmental and Behavioral Pediatrics, 33(5), 396-404. https:// doi.org/10.1097/DBP.0b013e318257fac3.

UNICEF. (n.d.). Coronavirus disease (COVID-19): What parents should know. https://www.unicef.org/stories/novel-coronavirus-outbreakwhat-parents-should-know. Accessed 15 April 2020.

UNICEF. (2020). Navigating pregnancy during the coronavirus disease (COVID-19) pandemic. https://www.unicef.org/coronavirus/ navigatingpregnancy-during-coronavirus-disease-covid-19pandemic. Accessed 20 April 2020.

UNICEF. (2020). Global Technical Assistance Mechanism for Nutrition, \& Global Nutrition Cluster. (March 30). Infant \& young child feeding in the context of Covid-19. https://www.unicef.org/media/ 68281/file/IYCF-Programming-COVID19-Brief.pdf. Accessed 10 April 2020.

United Nations Population Fund. (2020). UNFPA statement on novel coronavirus (COVID-19) and pregnancy. https://www.unfpa.org/ press/unfpastatement-novel-coronavirus-covid-19-and-pregnancy. Accessed 10 April 2020.

University of Saskatchewan. (2020). COVID-19 FAQs. https://medsask. usask.ca/covid-19.php. Accessed 5 May 2020.

Victora, C. G., Bahl, R., Barros, A., França, G., Horton, S., Krasevec, J., Murch, S., Sankar, M. J., Walker, N., \& Rollins, N. C. (2016). Breastfeeding in the 21st century: Epidemiology, mechanisms, and lifelong effect. The Lancet (British Edition), 387(10017), 475-490. https://doi.org/10.1016/S0140-6736(15)01024-7.

Wagg, A. J., Callanan, M., \& Hassett, A. (2019). Online social support group use by breastfeeding mothers: A content analysis. Heliyon, 5(3), E01245. https://doi.org/10.1016/j.heliyon.2019.e01245.
Wenham, C., Smith, J., \& Morgan, R. (2020). COVID-19: The gendered impacts of the outbreak. The Lancet, 395(10227), 846-848. https:// doi.org/10.1016/S0140-6736(20)30526-2.

World Health Organization. (2020a). Q\&A on coronaviruses (COVID19). https://www.who.int/news-room/q-a-detail/q-accoronaviruses. Accessed 25 April 2020.

World Health Organization. (2020b). Q\&A: Breastfeeding and COVID19. https://www.who.int/emergencies/diseases/novel-coronavirus2019/question-and-answers-hub/q-a-detail/q-a-on-covid-19-andbreastfeeding. Accessed 25 April 2020.

World Health Organization. (2020c). Infant and young child feeding. https://www.who.int/news-room/fact-sheets/detail/infant-andyoung-child-feeding. Accessed 25 April 2020.

World Health Organization (2020d). Breastfeeding advice during the COVID-19 outbreak. http://www.emro.who.int/nutrition/nutritioninfocus/breastfeeding-advice-during-covid-19-outbreak.html. Accessed 25 April 2020.

World Health Organization. (2020e). Frequently asked questions: Breastfeeding and COVID-19 for health care workers. https:// breastfeedingalberta.ca/wp-content/uploads/2020/04/faqsbreastfeeding-and-covid-19.pdf. Accessed 29 April 2020.

Zhu, H., Wang, L., Fang, C., Peng, S., Zhang, L., Chang, G., \& Xia, S. (2020). Clinical analysis of 10 neonates born to mothers with 2019nCoV pneumonia. Translational Pediatrics, 9(1), 51-60. https://doi. org/10.21037/tp.2020.02.06.

Publisher's note Springer Nature remains neutral with regard to jurisdictional claims in published maps and institutional affiliations. 